\title{
Marketing, estrategia y supervivencia: etología de la seducción*
}

\section{Marketing, strategy and survival: ethology of seduction}

\author{
Iván Fernando Castro del Río Ceballos**
}

Recibido: 17 de noviembre de 2017

Revisado: 13 de diciembre de 2017

Aprobado: 18 de enero de 2018

\section{Resumen}

El concepto de estrategia se toma como un camino que lleva al cumplimiento de objetivos y a alcanzar metas definidas que permiten a una persona o a un grupo sobrevivir y evolucionar en su espacio. Se ha definido a través de las disciplinas de la guerra, el mundo empresarial y el comportamiento de las personas. Existen estrategias para distintos escenarios en modelos económicos y empresariales, para ataques o defensas en tiempos de guerra, a fin de definir comportamientos de las personas y seducir. Pero también existe otro escenario en las estrategias, que presenta resultados de una forma despiadada

* Artículo de investigación producto del proyecto denominado: "Marketing darwinista: la etología en la seducción", adscrito a marketing y negocios internacionales de la Universidad Sergio Arboleda. Citar como: Castro del río , I. (2017). Marketing, estrategia y supervivencia: etología de la seducción. CIFE, 19(31), 157-175. DOI: https:// doi.org/10.15332/s0124-3551.2018.0031.06 
e inclemente en que participan los demás integrantes del reino animal, además del ser humano. Para el presente proyecto se hizo un análisis de animales depredadores, su comportamiento y la utilización estratégica que hacen del ambiente donde están para cazar y sobrevivir. Estos comportamientos en determinadas especies requieren una elaboración más específica y una coordinación especial con miembros de la misma especie o con elementos en su hábitat, procedimientos y habilidades dignos de considerarse como "estrategias de cacería", susceptibles de analizarse e interpretarse bajo el lente del marketing. En tal virtud se enseñan ejemplos de estrategias utilizadas por empresas que pueden ser comparables con las estrategias que utilizan algunos animales depredadores para cazar. A la vista de los resultados se concluye que la investigación en animales depredadores y sus estrategias para cazar puede ser una fuente enriquecedora para inspirar estrategias en las empresas.

Palabras clave: animales depredadores, empresa, estrategia, etología, marketing estratégico.

Glasificación JEL: M31, L1

\section{Abstract}

The concept of strategy is taken as a path which leads to the accomplishment of objectives and to reach defined goals allowing a group or a single person to survive and evolve in their space. It is defined through the disciplines of war, the business world and the people's behavior. There are strategies for different scenarios in economic and business models, for attacks or defenses in times of war, to define people's behaviors and seduce. But also, another scenario within strategies exists, one which presents results in a rough and visceral manner, where the other members of the animal kingdom act, besides the human being. A predatory animal's analysis was made, its behavior and the use they make of the environment where they are to hunt and survive. This behavior, in certain species require a more specific elaboration and a special coordination with same members of the specie or with elements in their habitat, procedures and skills worth to be considered as hunting strategies, strategies that can be analyzed and adapted under a marketing lens. Consequently, examples are shown about strategies used by enterprises which could be comparable with the strategies used by some predatory animals to hunt. In view of the results it can be concluded that the research in predatory animals and their hunting strategies could be an enriching source to inspire strategies in enterprises.

Keywords: enterprise, ethology, predatory animals, strategic marketing, strategy.

Glassification JEL: M31, L1 


\section{Introducción}

En esta investigación se identifican las estrategias nacidas de un comportamiento racional y elaborado, observado en animales depredadores. Si bien hay animales en cuyo honor se han nombrado algunas estrategias militares y de marketing, aún hay elementos por descubrir, sobre todo, en actitudes, trabajo en equipo, roles y labores específicas de miembros de un mismo grupo, no solamente en comportamientos simplemente instintivos.

Cabe resaltar que las estrategias por mencionar no son la única herramienta que usan o han usado los animales, pues las estrategias son procesos cognitivos y no solo un uso de características físicas o habilidades propias de una única especie. Así, es preciso definir el concepto de estrategia antes de hacer una observación eficiente del comportamiento animal, resolviendo cuáles pueden ser aplicadas e implementadas como estrategias de marketing.

La palabra estrategia proviene del griego strategia, que traduce "don de mando" y se conoce como "el arte de diseñar o emplear planes para alcanzar una meta" (Loftus, 2010). La estrategia se adaptó a las altas esferas de mando en el campo de la guerra, en donde el hombre explotó un inefable potencial en miles de procesos, ataques específicos, creatividad y elementos sorpresa para salir victorioso en cada batalla y hacerse con la gloria total en la guerra.

Esa prevalencia que buscaban quienes desarrollaban estrategias exitosas, analizaban a otros estrategas e incluso muchas veces copiaban el modelo, también se ve en otra dimensión en el mundo, donde existe el mismo instinto evolucionado de ir más allá de la supervivencia y hacerse un cómodo lugar entre los demás. En el reino animal también hay seres que desarrollan sus propias estrategias para conseguir alimento o para cuidarse de no ser el alimento de otros depredadores, alcanzando así un lugar preeminente en la cadena.

En la cadena alimenticia ${ }^{1}$ existe un orden dado por las capacidades, la fuerza, los atributos y los hábitos alimenticios de los animales. El modelo de la cadena alimenticia busca explicar que, por cada grupo de especies que se comen unos a otros, no necesariamente la fuerza física o las armas naturales letales que tengan, por evolución, les significa una victoria segura. En el mejor de los casos es una ventaja sencilla, que no promete nada.

Los animales que se van a analizar en este proyecto de investigación son los actores de segundo nivel ${ }^{2}$ dentro de la cadena alimenticia, es decir, los depredadores que se

1 La cadena alimenticia, o cadena trófica, es el diagrama en donde se explica la alimentación de los seres vivos, comenzando por quienes reciben su alimento de la energía del sol, luego los que se alimentan de estos y así sucesivamente.

2 Segundo nivel en la cadena alimenticia son las especies que se alimentan de especies no carnívoras, que se alimentan de plantas y organismos vegetales. 
alimentan de animales herbívoros y otros carnívoros de un menor tamaño, por lo general. Estos actores son una gran fuente de estrategias de cacería, no necesariamente son las especies que cazan en grupo, ni los de mayor tamaño.

Desde una óptica empresarial, las instituciones están perdiendo su sentido de persuasión, de orientación que se desarrolla en marketing ante el consumidor (Klaric, 2016). Si el consumidor decide de manera impulsiva y no racional, ¿qué sentido ha tenido el hecho de suministrar recursos a diario para comunicar a las personas que un producto es mejor que otro, que trae más beneficios, incluso que es más económico?

Las ventas tienen su propio motor, que tiene que ir ensamblado dentro de marketing, pero no son un resultado único y transparente de lo que está sucediendo. Las ventas son una consecuencia del proceso de marketing. Bajo esta consideración no tiene sentido hacer que una persona racionalice sus compras. En ese caso, el producto que valga menos o el que traiga más contenido tendría una ventaja sobre el resto, indiscutiblemente (Edwards y Day, 2010). La emocionalidad es el factor que determina lo que se refiere a tomar acción, la racionalidad es lo reflexivo. Como cuando una persona piensa demasiado qué debió haber dicho o cómo debió haber actuado frente a una situación que ya pasó y sobre la cual ya no tiene algún poder de decisión. El marketing mismo es seducción.

\section{Marco teórico}

Existe un comportamiento que asumen los vendedores y que promueven por efectos de motivación y de persecución de metas, logros y objetivos. Un comportamiento que asumen frente a los que son sus compradores habituales, potenciales o anteriores; son sus clientes con quienes deben negociar día a día y quienes también se preparan para la negociación. Los vendedores asumen un rol de cazador y se entrenan bajo una filosofía de acecho, en donde hay que acercarse sigilosamente para que el comprador (su presa), no se escape ni se asuste. La intención de vender al primer contacto es considerada como un error. Otros llevan el ejercicio un paso más allá y se toman el papel más en serio, se confunden entre el ambiente en donde se mueve el comprador, se acercan despacio o esperan el momento justo para lanzar su oferta, momento en que para el comprador negarse sea forzoso.

Luego vino una evolución desarrollada por Levitt (1960), señaló los problemas que traía el hecho de enfocarse en la transacción, quedándose en el producto y no mirando al consumidor o al mercado; así las compañías desarrollaron marcas y productos pensando en lo mucho que les gustaría a sus compradores en el presente y para el futuro. Muchas lograron impactar y fascinar, pero la gran mayoría se perdía en el camino (Levitt, 1960). 
A lo largo de los años la estrategia militar se ha adaptado y puesto al nivel de la vida empresarial, de los negocios bilaterales o multilaterales. La política, las relaciones con las personas y la vida cotidiana, se han comparado con un campo de batalla. En Greene (2011) se comparan a cada uno de ellos con la guerra, en donde también se necesitan estrategias que persiguen una victoria. El autor afirma que en esos verdaderos campos de batalla se exige contar con tácticas, maniobras y planes eficaces.

Por otro lado, existe un mundo aún no tan explorado a fondo como el de la vida militar, en el que las estrategias no se han adaptado a productos, precios, personal, comunicación, distribución o compañías. En el reino animal abunda la necesidad de supervivencia, de proveer a una familia, de cazar, de no ser cazado y de mantener lo que se ha conseguido. Frente a este y muchos otros tipos de requerimientos y necesidades, los animales se han visto también forzados a ir más allá de su comportamiento instintivo, numerosos estudios destacan especies día a día por su inteligencia, descubren lo que se presume, debería ser obvio desde un principio, ya que todos los seres están amparados bajo la misma regla natural de evolución, en donde no existe otro camino más que el de avanzar para hacerse un lugar en la vida, en el mundo (Darwin, 1859).

\subsection{Breve revisión de la literatura}

La credibilidad de la marca siempre ha jugado un papel muy importante en el mercado, se trata del prestigio y el buen nombre que posea una empresa y un producto frente a los consumidores. La marca debe tener personas que crean en ella y sea ulterior a los colaboradores. Es decir, pese a que se cambie al personal, las creencias y convicciones de la marca deben permanecer intactas. La creencia que genera la marca es la forma en que esta se toma al mundo, tiene que existir un vínculo inasible pero fuerte entre la creencia y la capacidad de la marca. Es la forma de contar de qué manera la marca hace su mejor esfuerzo de visibilización. No tiene caso que la marca realice promesas o elabore creativos slogans que generen un atractivo emocional en las personas, si a la larga no se cumple una sola palabra de lo que se dice (Edwards y Day, 2010).

Estudios sociales en la última década sobre diferentes marcas alrededor del mundo, desde Procter and Gamble hasta la Federación Brasilera de Fútbol, indican que las personas en el mundo actual valoran los vínculos sociales que crean los productos más que a los productos mismos. Lo que se requiere es la comprensión de que el valor de conexión de un producto o servicio resulta solamente de personas interactuando entre sí y que en el área de marketing y publicidad, los canales basados en eventos y experiencias son reglas que hay para reunir consistentemente a una audiencia para forjar interacciones relevantes y emocionantes entre ellos. La multitud es el elemento más importante de la experiencia (Lyons, 2017). 


\subsection{Las estrategias}

El núcleo de la guerra se define como un acto de fuerza que se lleva a cabo para obligar al adversario a acatar ciertos preceptos determinados por la fuerza, para enfrentarse a otras fuerzas contrarias, recurre a las creaciones de la ciencia y el arte, donde el objetivo es la imposición de la voluntad propia ante el enemigo y para llegar a ese fin hay que desarmarlo (Clausewitz, 2005).

El General Von Clausewitz se refería a la fuerza inicial como algo meramente físico, en donde se emplean recursos de una nación para alimentar esa fuerza y hacerla más imponente que el resto. En el mundo empresarial el concepto no se aleja tanto: la fuerza física de una empresa resulta ser sus recursos financieros, el conocimiento único junto con los procedimientos que maneje, las marcas y patentes que tenga registradas, entre otros elementos. Los soldados siguen siendo las personas que representan a la firma y llevan a cabo la estrategia planteada.

Los esfuerzos realizados por los soldados, que ejecutan la estrategia de su líder, quien a su vez es quien responde ante los altos mandos por las bajas, errores o aciertos presentados durante la operación, se dan con base en el fin último que es la supervivencia. El general Eisenhower reconoció la necesidad de improvisar mientras se ejecuta un plan, necesidad de confiar en el buen trabajo de los demás y de confiar en el instinto mismo, como lo hacen los animales (Loftus, 2010).

El comportamiento denotado por los animales, empleado en la necesidad que tienen de sobrevivir y comer, sobresale con los métodos que utiliza cada especie para lograr su objetivo de atrapar a su presa. (Timbergen, 1951) definió los niveles en que la etología investiga el comportamiento: Evolución, función, causación y desarrollo. La etología cuenta con distintos enfoques gracias a los aportes que han realizado diversos investigadores a lo largo de la historia, uno de los más célebres fue Konrad Lorenz, en honor a quien se nombra la Etología Lorenzana, que se refiere a un comportamiento bajo el prisma evolutivo de las especies. Estos enfoques a menudo hacen dudar a los científicos sobre nombrar sus investigaciones como Etología o como Biología comportamental (Taborsky, 2006).

Timbergen definió de manera simple a la etología como la biología comportamental, no hay necesidad de utilizar un término en lugar del otro porque no hay diferencia alguna entre ellos, para hacerlo más completo quiso desarrollar el estudio comportamental de los animales bajo los cuatro pilares mencionados anteriormente, que intentan explicar, en principio, de dónde puede haber surgido ese comportamiento y si hay un patrón común en los comportamientos animales (evolución); si el comportamiento es inducido o causado (causación), en qué beneficia al animal este comportamiento (función) y qué 
consecuencias inmediatas o tardías acarrea o requiere este comportamiento en el organismo propio (desarrollo) (Timbergen, 2005).

Desde la perspectiva del marketing es preciso definir los conceptos para poder relacionarlos e interpretarlos en términos de mercado. Se conoce al cazador como la marca, pero la presa no es una persona en específico, no es el cliente; tampoco es un grupo de personas, la presa no es el segmento. La presa en marketing es un concepto, es el concepto más enriquecedor de todos, de hecho, es la demanda.

A continuación se hace un análisis de diferentes estrategias que utilizan algunos animales depredadores en el mundo para cumplir su objetivo de capturar a la presa, se hará una descripción de la estrategia que usa el animal, posteriormente viene la observancia de la estrategia, en donde se explica la aplicabilidad de esta en Marketing y finalmente se dará un ejemplo de una empresa que haya tenido éxito siguiendo una estrategia similar a la planteada.

\section{El carrusel de la Orca}

La Orca, o ballena asesina, es uno de los animales más inteligentes conocidos por el hombre, pariente de los delfines y que tiene un lenguaje propio; es el primer actor del análisis de estrategias. A lo largo de las observaciones científicas que le han hecho a la especie los biólogos de todo el mundo, existe una estrategia específica que fue bautizada como el carrusel, que básicamente se trata de un grupo de orcas, coordinadas entre sí, que, en lugar de lanzarse con la boca abierta para engullir la mayor cantidad de peces, hacen de perros pastores guiando un rebaño, nadan alrededor impidiendo que los peces toquen el fondo y logrando que formen una esfera.

La sofisticación de la estrategia viene cuando entre las orcas se turnan para dar vueltas por debajo de la esfera de peces, una tras otra en una perfecta coordinación. Cuando están en ese movimiento muestran su panza blanca, sueltan burbujas de aire y emiten sonidos, que aterrorizan a los arenques y hacen que la esfera se compacte aún más. Cuando las orcas aumentan su velocidad, esto hace que los arenques salten a la superficie en un intento desesperado por escapar, parece que el agua está hirviendo. En cuanto la manada tiene controlados a los peces, una orca golpea el borde del cardumen con la cola, dejando arenques inmovilizados, entre muertos y aturdidos y permitiendo a su manada comer (National Geographic, 2015).

\subsection{Observancia de la estrategia}

Cuando se va a traducir esta estrategia al mercado, hay que concebir a la marca como la manada de orcas. Se toma entonces la acción de mantener a los peces en una esfera 
gigante como la creación de la expectativa entre los consumidores, las orcas que nadan al lado son los mensajes y la comunicación que llega desde la compañía, envolviendo el ambiente con misterio y jugando con la fascinación de las personas, también es el esparcimiento de una cultura novedosa que pretende educar a los potenciales consumidores, en el caso de sacar un producto nuevo o muy diferenciado, estos mensajes normalmente son muy ambiguos y no muy claros, lo suficientemente atrayentes para generar un eco, para producir preguntas, incluso algunas veces, ni se mencione a la marca, ni lo que pretende.

Después viene la orca que va por debajo, enseñando el vientre, haciendo ruido y soltando burbujas de aire, este ya es el mensaje directo, una promoción escandalosa y una serie de comunicaciones que despierten un interés directo del consumidor, el cual sin embargo se encuentra aún indeciso de si involucrarse o no en el proceso con la marca.

Finalmente, el coletazo. Un coletazo es una táctica contundente que permita el flujo natural y esperado de la demanda, en términos técnicos de mercado, el coletazo es lo mismo que el detonante. Puede ser cualquier cosa lo suficientemente irresistible como para asegurar el éxito de la marca y su proyecto. Puede abarcar desde una asociación estratégica con otra marca hasta un cambio ligero en el empaque.

\subsection{Aplicación de la estrategia}

En 1974 Nestlé compró el derecho para comercializar el diseño de un nuevo sistema para hacer café, elaborado por científicos en Suiza. Ese fue el principio de lo que hoy se conoce a nivel global como Nespresso.

La máquina era una revolución, era más compacta, sencilla y limpia que las otras cafeteras pesadas y frágiles, que además requerían el toque especializado de expertos para hacer el café. El nuevo sistema de Nespresso ofrecía el concepto de hacer un expreso de la manera más personalizada posible, desarrolló una variedad de sabores en varias presentaciones, todas en distintos empaques de aluminio. Sin embargo, Nespresso resultaba revolucionario hasta para las mismas creencias de la empresa, pues construir una marca en torno a una máquina y no en torno a productos empacados transgredía la manera en que la mayoría de los ejecutivos de Nestlé estaban acostumbrados a actuar.

Después vino una reorientación dirigida por un director nuevo, contratado para manejar el proyecto Nespresso, que, a pesar de su tecnología y su prominente facilidad para el consumo, no parecía tener un punto de arranque. En 1988 llegó Jean-Paul Gaillard a redefinir el producto y reorientarlo a los hogares donde las amas de casa adineradas y sofisticadas apreciarían infinitamente este sistema, que sería un alto grado de satisfacción para su gusto por el café gourmet. Los ensayos en las pruebas de mercado y los datos de encuestas arrojaron una demanda potencial modesta y que un precio que 
trazaría la demanda era uno mayor a los 0,25 céntimos suizos $(0,16$ centavos de dólar, aproximadamente).

La lección que Nespresso le estaba dejando a sus padres creadores y al resto del mundo era que un producto innovador no es suficiente para generar gran demanda. Las ventas en el mercado de los hogares comenzaron a un ritmo muy lento, la mayoría de los almacenes no confiaban en tener en su inventario esas máquinas raras y a los que aceptaban no les interesaba tener disponibles las bolsitas de cafés surtidos. El cardumen se estaba dispersando más y más, Nespresso no estaba produciendo ni simulando una temperatura lo suficientemente alta como para mantener la esfera compacta.

La solución para evitar que el cardumen se dispersara fue la creación de un cuestionado club de Nespresso, cuyo objetivo principal era la venta directa de las bolsitas de café a los hogares, eliminando intermediarios en la cadena de distribución y con la espera de dos días, en la puerta de las casas de los miembros del club Nespresso. El club recibió el primer día tres inscripciones, el segundo once más y el tercero, ninguna. Los días fueron semanas, las semanas meses y de repente empezaron a llover inscripciones, de decenas, centenas más adelante. Para 1990 el club Nespresso contaba con 2700 miembros en Suiza, Francia, Japón y Estados Unidos. Esta estrategia de implementar el club fue el aumento de velocidad que logró compactar al cardumen.

La venta directa es una garantía de la frescura del producto y unos estándares de servicio gradualmente altos. Nespresso tenía una línea telefónica donde hacía recomendaciones y ofrecía información 24 horas al día, luego fue en línea. Este servicio tan magnífico para los consumidores daba paso a uno de los fenómenos más grandes en la creación de demanda: el marketing viral. Cada persona recomendaba y refería personas hacia Nespresso y hacía que la red se expandiera más y más como un globo. Sin embargo, todavía faltaba el coletazo definitivo, el que sellara el destino del cardumen.

El coletazo resultó ser muy poderoso, se refirió a la posibilidad de hacer que las personas degustaran el producto, integrando su máquina en las aerolíneas, que servirían el superior Nespresso a sus pasajeros. La primera aerolínea en servir Nespresso fue Swiss air, otras siguieron el ejercicio y las ventas de la máquina comenzaron un ascenso prudente. El segmento de pasajeros de primera clase en una aerolínea, ricos, sofisticados y acostumbrados a viajar era el segmento perfecto para Nespresso. Prontamente las demostraciones de Nespresso se trasladaron a los restaurantes más sofisticados en países europeos, hubo líderes de opinión seleccionados, como periodistas y políticos, a quienes se les obsequió una cafetera Nespresso, para sus invitados VIP. El crecimiento de las ventas de Nespresso mejoró. Ubicar a Nespresso en las aerolíneas fue el coletazo que necesitó Nestlé para sellar el destino del cardumen y alimentarse como realmente merecía (Slywotzky, 2012). 


\section{La exploración del zorro rojo}

En los terrenos cubiertos de nieve, de varios pies de alto, el zorro rojo se dedica a capturar a su presa de la manera más sorpresiva y exacta, incluso, cuando las presas se encuentran fuera de la vista de él mismo, puede detectar su locación exacta. Generalmente las presas son roedores de menor tamaño que transitan por debajo de la tierra o de la nieve.

$\mathrm{Al}$ detectar la posición de su presa, el zorro salta de una manera única y particular sobre ella, se sumerge de cabeza bajo la nieve y al poco tiempo después de haber saltado, emerge victorioso con la presa entre sus dientes.

$\mathrm{Al}$ estar por encima, sus presas jamás lo ven venir. Pero de todas formas resulta formidable que solo por sonidos se puede detectar la locación de sus objetivos, que no haya algún factor adicional que impulse de mayor manera el propósito del zorro. Una investigación de dos años realizada por Cerveny en la República Checa, en donde se observaron 84 zorros distintos y más de 600 saltos, reveló un componente extra que ayuda a la orientación del zorro conforme a su objetivo, pues cuando los zorros saltaban en dirección noreste, sus ataques tuvieron un $73 \%$ de acierto. En otras direcciones, los resultados positivos se disminuyeron dramáticamente. El zorro se vale entonces de la combinación de su agudo sentido del oído y del campo magnético de la tierra para localizar a su presa de manera exacta y poder atraparla.

El zorro usa el campo magnético de la tierra a manera de telémetro, busca el punto exacto en donde el ángulo del sonido concuerda con la pendiente del campo magnético. Así sabe qué tan lejos debe saltar para estar sobre la presa y atraparla. Los tiburones, los pájaros, las hormigas y las vacas son capaces de sentir los campos magnéticos, aunque ellos usan esta habilidad para determinar su propia locación o dirección (Moss, 2013).

En resumidas palabras, el proceso dice que los zorros rojos se concentran profundamente usando su sentido del oído, lo combinan con su habilidad para sentir el campo magnético terrestre, luego de rastrear a su presa y detectar su locación exacta, calculan el punto y el momento en que hay que abalanzarse sobre ella, y tienen éxito siete de cada diez veces que lo hacen.

\subsection{Observancia de la estrategia}

$\mathrm{Al}$ ser esta una estrategia que involucra rastreo, detección de posiciones e incluso una persecución en algunos casos, en marketing esto tiene que ver con el uso apropiado de la investigación de los mercados, en donde la exploración al consumidor, la búsqueda de alternativas y de información en general, es el método esencial a la hora de tomar decisiones claves. 
En primera medida, usar el sentido del oído para detectar presas debajo del suelo o de la nieve puede traducirse a la búsqueda de tendencias y de mercados que pueden considerarse como potenciales, nuevos caminos, pruebas que estén realizando los competidores (si es que acaso se llega a tener acceso a esta información), comportamientos o cambios leves en los márgenes de rentabilidad de algún producto o servicio, incluso el mismo posicionamiento y el comportamiento de las personas frente a él, puede ser objeto de escucha y olfateo en esta etapa.

La segunda etapa es el uso del campo magnético y la combinación con el oído para detectar y calcular la posición de la presa. Dimensionando esta segunda etapa en marketing, se refiere al análisis y el uso del entorno, pero no solo como observación o un análisis concluyente mirando los números que arrojan las estadísticas, en esta etapa hay que sacar provecho de la capacidad de sentir el mismo campo magnético, esta capacidad se traduce en envolvimiento, el análisis en primera persona de la investigación por parte de la marca. Las investigaciones etnográficas son las que más se han acercado a este tipo de análisis. Las palabras clave en esta etapa son "involucramiento" y "envolvimiento". En este punto es donde se explota la capacidad de la marca para conectar con la gente, en su etapa de investigación.

El paso final es usar la información obtenida durante las dos primeras etapas para lanzar un plan de acción, un procedimiento que permita la consecución de los objetivos y deje a la marca un paso más delante de la competencia, le proporcione un aire de innovación y le abra paso a la compañía para liderar el mercado. El tercer paso es abalanzarse sobre su presa, sobre la demanda.

Este paso puede involucrar esfuerzos y cantidades extras de trabajo que puede que no hayan sido contempladas previamente, como enfrentamientos directos con la competencia, es el llamado a la acción. También puede traer dificultades que no estaban antes ahí y se formaron por acción de la marca. En esta etapa hay que elaborar tácticas y maniobras de rápida ejecución para superar obstáculos, así como el zorro se lanza de cabeza y pretende llegar lo suficientemente bajo para luego emerger victorioso y honrar el $73 \%$ de efectividad en sus ataques, la marca tiene que estar dispuesta a trabajar de más para alcanzar el objetivo.

\subsection{Aplicación de la estrategia}

Los eventos se presentan en el vasto continente de África, en donde había una marca extranjera de cerveza que ya era conocida (año 1998), una marca respetada por muchos y se asemejaba a otras tónicas africanas tradicionales por su color y su amargor. La marca de la cerveza es Guinness y su objetivo era capturar el amor del público, dirigirlo hacia la cerveza para luego poder aumentar automática y significativamente sus ventas. La meta era duplicar las ventas para el 2005. 
Los mercados más grandes los representaban los países de Nigeria, Ghana, Camerún y Kenia, seguidos por otros de menor tamaño como Costa de Marfil, Gabón, Benín, Togo, Uganda, Tanzania y Burkina Faso. El mercado de mayores dimensiones lo traía Nigeria, que a su vez es el país más populoso de África con 160 millones de habitantes que tiene muchos retos en su economía, comenzando por el ingreso per cápita que es inferior a $\mathrm{U} \$ 3.000$. Su estructura era rudimentaria en 1998 y muy pocas personas tenían televisores, además que el acceso a contenidos de entretenimiento era escaso. Pocos africanos eran íconos en el cine o la televisión.

En este punto, Guinness realizó una investigación de la cultura africana, de sus costumbres, sus aspiraciones, pero no para traducirlas a números y luego moldear esos números en estrategias calculadoras y frías. En realidad, el enfoque fue saber qué sentía la gente por ser ella misma, qué se sentía ser africano, qué significaba provenir de Nigeria y qué estaban dispuestos a decirle al mundo. El primer movimiento de la marca fue la introducción de un personaje que resaltara el orgullo y las cualidades de grandeza del hombre africano, se creó a Michael Power: una especia de agente secreto que luchaba contra villanos y siempre salía victorioso, honraba a la marca Guinness y más que todo representaba todo lo que se quería ser en África, que los hombres podían ser admirados y alcanzar también la grandeza, fue un símbolo de esperanza y de inspiración para millones de personas. Esta campaña logró tener la larga vida de siete años, el reconocimiento de marca en países clave alcanzó un $95 \%$ y el de Michael Power fue de un $93 \%$ en todo el continente. A dos años del 2005 se cumplió la meta que habían propuesto de duplicar las ventas.

De todas formas, el amor del público africano todavía no estaba ganado del todo por la marca, el paso siguiente fue seguir explorando para generar más involucramiento y poder entender al público, poder identificarse con ellos y buscar conectar con ellos al final. En este punto, la agencia a cargo de hacer la investigación para Guinness, Saatchi y Saatchi, usó un arma de su propio arsenal, una metodología de investigación etnográfica que ellos mismos denominaron "Xploring" que se encargaba de cazar insights significativos de los consumidores, fue una decisión acertada. Los resultados que arrojó la investigación hablaron de la aceptación en África que la vida era invariablemente dura y que las personas encontraban una inspiración en cualquier cosa, que impulsaba el deseo de elevarse por encima de las circunstancias y que la posibilidad de éxito estaba a la vuelta de cualquier esquina.

Esta vez, a lo que Guinness apeló fue a dos ejes claves en la campaña, que eran la "grandeza" y "creer", siguiendo este enfoque sacaron la inspiradora campaña de Udeme, un hombre que soñaba con trabajar en el cielo y al final resultó piloteando un avión que entregaba suministros, la campaña resultó con tanto éxito que la gente llegó a apodar a la cerveza "Udeme”, y al brindar decían "ique la cerveza vea el cielo!”. 
En 2011, la campaña que siguió con la línea emocional de contar historias que buscaran inspirar a quienes las recibían fue esta vez dirigida al enfoque del jefe de la familia y el fútbol. La campaña de "mi hermano mayor Femi" contaba una historia sobre crecimiento, la aspiración a conquistar otros lugares y volver para ser el jefe del hogar, fue un nuevo peldaño en el camino de inspirar a la gente, uno exitoso. La gente no vio un comercial, no vio una publicidad sino vio la historia de dos personas reales.

La demanda en ese proceso entero fue la representación de una exitosa seducción. La estrategia del zorro rojo, en donde se pretendió oír a las personas y lo que hacían, identificarse con ellas, conectar a la marca y volverla una más de la población y finalmente abalanzarse sobre la demanda, convirtiendo a Guinness en una lovemark, es el resumen de trece años de investigación y planeación estratégica.

\section{La honda sin retorno de la Naatlo Splendida}

La Naatlo Splendida es conocida como la araña "slingshot" o "tirachinas", por su habilidosa manera de atrapar insectos con su telaraña estirada. Tiene una habilidad impresionantemente efectiva para atrapar insectos, sobre todo insectos con alas y en pleno vuelo.

El procedimiento parece simple, la araña prepara su pegajoso telar, coordina el movimiento hilo tras hilo entre ramas y hojas de árboles y cuando los insectos vuelan en el rango de su visión, estando en el centro de su telaraña, la estira hacia atrás formando un cono y luego la lanza sobre la presa. Sucede en cuestión de segundos, el insecto no tiene a dónde escapar y podría decirse que desde que la araña lo vio con sus diminutos ojos, el insecto no tenía oportunidad alguna.

La araña tiene que haber seleccionado el lugar donde existe una afluencia aceptable de insectos, haber observado la trayectoria de estos al pasar por ese lugar y calculado el ángulo de inclinación necesario para que su lanzamiento sea efectivo, antes de haber comenzado a tejer su red. Luego de esto, podría decirse que la acción de caza condensa la preparación minuciosa y efectiva de la red, la detección del objetivo y el lanzamiento hacia él. Todo perfectamente orquestado y con la mayor velocidad posible, la capacidad de reacción para la araña tiene que ser mínima, pues mide menos de un centímetro y caza presas muchísimo más grandes que ella. Pero también mucho menos preparadas. (National Geographic, 2010)

\subsection{Observancia de la estrategia}

Esta estrategia que involucra redes perfectamente coordinadas, orquestadas y a una velocidad muy notable puede ser igualada en el mundo del marketing con el sistema que 
se encarga de la distribución, la cadena de abastecimiento es la estrategia que se iguala a la de la talentosa Naatlo Splendida.

El primer paso es la coordinación exitosa de una red de distribución, preparada para responder con una demanda esperada, este tipo de estrategia es recomendable que se maneje con marcas que ya tengan experiencia en el campo de la distribución, la producción acelerada y los procesos como el fust in time.

El segundo paso viene a ser el público, este tipo de estrategias debe ser más push que pull, trata siempre directamente con el cliente, promoviendo una gran celeridad en el ejercicio de mercado. Una manera efectiva de lograrlo es, por supuesto simplificando la cadena y eliminando intermediarios, no está de más advertir en este proceso que la calidad del producto o el servicio puede verse sensiblemente comprometida.

El tercer paso es el llamado a la acción, la interacción y la puesta en marcha, los tres pasos vienen muy seguidos, los periodos de tiempo entre las etapas son, de una u otra manera, inexistentes. Lo que se quiere generar con esta estrategia es un impacto de gran volumen entre la competencia, el consumidor y el público presente en general. La inversión en este tipo de estrategias suele ser muy elevada, al igual que el riesgo. Pero una vez implementada, el éxito de una estrategia de esta clase garantizará un posicionamiento para la marca y una fascinación y aceptación masiva por parte del público.

\subsection{Aplicación de la estrategia}

El mejor ejemplo que se puede nombrar en este tipo de estrategia es uno que involucre una industria dinámica, altamente exigente y demandante en términos de creatividad, producción en gran volumen y sobre todo la capacidad de cautivar a las personas con conceptos. El ejemplo viene de la industria de la moda.

La alineación que presenta Zara en su sistema de producción y el de distribución no presenta ninguna escisión. Y justamente eso es lo que se necesita, era preciso un sistema que pudiese con la tarea de introducir semanalmente prendas nuevas y reponer las colecciones existentes. Este sistema tenía que ser ágil y eficaz. Inditex en consecuencia, dotó a cada una de sus cadenas de centros de distribución donde se realizan envíos diferentes a las tiendas por semana (Martínez, 2012).

Las tiendas de moda por lo general cuentan con un patrón de sacar una colección por cada temporada, las más grandes se dan el lujo de hacerlo dos veces al año, en primavera y verano, otras más veces y siempre relacionando sus confecciones y su estilo con las estaciones. Esta práctica genera expectativa y ansias en el público, lo que al final se traduce en volúmenes de ventas en el momento que sale la colección y en ciertos casos, fidelización. 
Lo que hace Zara es completamente distinto, fue revolucionario en sus inicios y aún hoy lo logra ser, pues no juega con la expectativa del consumidor, no pregona qué va a venir la siguiente temporada, sino que pasa a la acción de inmediato, apelando al factor sorpresa en los consumidores, lo cual es gratamente recompensado. De todas formas, a nadie le gusta sentir que lo están haciendo esperar adrede.

Zara resume la estrategia de la Naatlo Splendida honrando la celeridad de sus procesos, la estructura en sus redes de producción y de distribución es impoluta, el objetivo ya está claro, pues son sus clientes habituales y personas que simplemente entran a la tienda a ver qué hay de nuevo, para siempre salir con una sorpresa y una bolsa negra que dice Zara colgando de sus dedos. Pues el tercer paso es el llamado inmediato a la acción, el cumplimiento de los deseos de las personas, sin que haya pedido alguno. En este tercer acto, Zara suelta su elástica telaraña y logra atrapar la demanda.

\section{Materiales y métodos}

$\mathrm{Al}$ analizar el escenario de la estrategia y su aplicación en la guerra, la empresa, la vida cotidiana de las personas e incluso la cacería por parte de algunos animales depredadores, se pretendió profundizar lo último y llevarlo a un escenario de mercadeo, intentando descifrar de qué manera cabría cada estrategia analizada en los distintos ámbitos del marketing, desde el producto hasta la comunicación de la marca.

La perspectiva que otorga este nuevo análisis requiere de una interpretación novedosa, de una mente abierta al cambio y cierta capacidad relacional. Se buscaron opiniones de expertos en el campo del mercadeo en empresas y universidades para validar estas aplicaciones de estrategias tomadas de algunos animales depredadores y sus particulares formas de conseguir su supervivencia y la de los suyos.

Se buscaron pues opiniones de decanos de facultades de marketing en distintas universidades de Bogotá y de directores o jefes de mercadeo de varias empresas, quienes compartieron sus opiniones al respecto, debatieron sobre la posibilidad de esta nueva perspectiva en el campo del marketing estratégico y reflexionaron ante la posibilidad de generar más investigación en los animales y su comportamiento, para aplicarlo a la vida empresarial.

\section{Discusión}

Todos los entrevistados definen la presencia de un objetivo, de una meta para que la estrategia tenga sentido y existencia. Se les dice logro de resultados, objetivos y marco 
temporal definidos o finales deseados. La estrategia incide en marketing de distintas maneras para los decanos y para los empresarios; para los empresarios la estrategia incide en las maneras que hay de escuchar al mercado para partir de ahí, para los decanos la estrategia tiene que ver con la empresa, con la capitalización de las oportunidades del mercado y la estructura organizacional.

Los decanos conocen y le tienen más familiaridad a estrategias teóricas clásicas como las matrices BCG, ${ }^{3}$ Ansoff, ${ }^{4}$ estrategias de teóricos como Porter, Kotler y Drucker, modelos estratégicos como el modelo $\mathrm{STP}^{5}$ y también estrategias de marketing social. Los directores de marketing de las empresas cuentan con modelos menos teóricos y prefieren los que generan resultados e invitan a la acción con una mayor celeridad. Entre estas están las matrices de McKinsey, ${ }^{6}$ los modelos Canvas $^{7}$ y las estrategias de ventas o para los diferentes procesos de la empresa.

Todos los entrevistados coinciden que una fuente de inspiración para las estrategias de marketing en una empresa debe ser el mercado, las personas, así como la información que proveen es un recurso que debe ser explorado por las empresas para lograr tener un enfoque acertado y certero frente a la satisfacción de necesidades y la generación de clientes. También coinciden los entrevistados en que el comportamiento animal puede ser una fuente provechosa que nutra las estrategias en una empresa; varios de los entrevistados no habían considerado la opción hasta el momento en que la pregunta les fue formulada. Sin embargo, reconocen el potencial y la riqueza que puede tener un análisis del comportamiento animal.

La disposición de los entrevistados frente al desarrollo de información sobre estrategias de animales aplicables al mundo empresarial fue absoluta, es un tema que a todos los pareció potencialmente provechoso e interesante, además de disruptivo e innovador.

3 Matriz Boston Consulting Group, que categoriza los productos según su comportamiento en demanda, accesibilidad y costos de producción; puede ser estrella, vaca lechera, niño problema o perro.

4 La matriz Ansoff es también conocida como vector de crecimiento, tiene ejes de productos nuevos o tradicionales vs mercados nuevos o tradicionales, creando cuatro cuadros en donde se ubica la acción a realizar según el escenario, puede ser penetración en el mercado, desarrollo de productos, desarrollo de mercado o diversificación.

5 Modelo de Segmentación, Targeting y Posicionamiento, es una estructura que facilita definir un enfoque frente al mercado, pretendiendo identificar a quién se quiere llegar, conocer más al público objetivo y cómo se quiere ser percibido por él.

6 Matriz desarrollada a principios de los años 70, es una matriz de nueve celdas en dos ejes, donde uno representa el grado de atractivo de mercado (bajo, medio, alto) y el otro la fortaleza de una unidad de negocio (baja, media alta).

7 Modelo Canvas es un modelo de negocio en donde se pretende identificar cada factor importante en una empresa, desde el factor que recibe los ingresos hasta el factor de los aliados claves del negocio. 


\section{Conclusiones}

El sector de la academia demostró que en lo que se refiere a la estrategia, se tiene en consideración el largo plazo y se dejan de considerar muchos factores internos, pese a que se intenta compilar todo, se habla de una manera muy general de la estrategia.

El sector empresarial habla de estrategias que muestren resultados en el corto plazo, estrategias más para reaccionar al mercado y no para producir demanda. Considera que lo dicho por los teóricos está bien para los libros pero que muchas veces son estrategias que no se saben ajustar a la realidad de una empresa y los requerimientos que tiene, en cuanto a tiempo y rapidez en producir resultados.

Todos los entrevistados reconocieron la falta de información y de desarrollo sobre el tema, lo cual lo hace potencialmente provechoso, tiene mucho que ofrecer al mundo empresarial y, pese a que una estrategia no es una fórmula mágica, puede servir de guía o referencia para situaciones particulares de mercado.

Las estrategias basadas en comportamiento animal pueden tomar caminos muy diversos, al estar sujetas a la interpretación de quien realiza el análisis; de cualquier forma, una estrategia tiene que ser diseñada frente a una situación particular. Esto no significa que las estrategias analizadas y presentadas en este trabajo no sean útiles, todo lo contrario. Son una guía y un camino que enseña a buscar inspiración en otras fuentes no tan convencionales.

Una interpretación que se le puede dar a las estrategias basadas en comportamientos con animales es de utilizarlos muy literalmente, como un símbolo para las marcas y dar mensajes extra en la comunicación con el consumidor, en las entrevistas se obtuvieron varios ejemplos donde se utilizaban animales de una forma muy puntual y simbólica, en lugar de pensar en ejemplos de estrategias usadas por ellos. Se mencionó al águila por diferentes personas y cómo era utilizada por países para representar valores en una nación; se mencionó también a las mariposas y su delicadeza explotada como símbolo en las joyerías.

$\mathrm{Al}$ mencionar aquellos modelos estratégicos que se conocían, los académicos hablaron de personajes teóricos referentes en Marketing, como Phillip Kotler, Michael Porter, incluso Peter Drucker, también se mencionaron matrices, como la BCG o Ansoff o el modelo STP. Sin embargo, en las empresas, los directores y cabezas de marketing hablaron de tipos de estrategias, enfoques de una estrategia y matrices de análisis al consumidor, pero no se mencionó a los teóricos como referentes en una estrategia. Incluso se dijo que una estrategia como las que plantea Porter es demasiado soñadora, demasiado rigurosa y no encaja con los tiempos ni ritmos que lleva el marketing. 
Las estrategias basadas en animales depredadores son un mundo inexplorado todavía, dispuesto a proveer con inspiraciones nada convencionales y potencialmente efectivas. Es preciso aumentar la investigación en este campo y fortalecer el conocimiento que se tiene frente a lo que hacen los animales en su entorno para sobrevivir, muy probablemente hay especies que al igual que la raza humana, han seguido una línea de evolución que vale la pena reconocer.

Las estrategias resultan ser un modelo de guía, no son un manual riguroso por seguir con pasos específicos y exactos que auguran un éxito sólido y resultados favorables en el corto plazo. Las estrategias mencionadas son a largo plazo, los ejemplos dados, son verídicos y mostraron sus resultados a largo plazo.

Por otro lado, los resultados no se deben medir simplemente en ingresos, no se debe buscar solamente aumentar el flujo de caja o incrementar los ingresos en una empresa, se debe tener un objetivo más integral. Guinness pretendía ser una Lovemark; Nestlé quería que la gente se adaptara a Nespresso y lo consumiese regularmente; Zara quiere que su marca sea altamente diferenciada de la competencia y valorada por quienes asisten a sus tiendas, esto muestra la integralidad de los desarrollos en el mercadeo moderno.

\section{Referencias}

Clausewitz, C. v. (2005). De la guerra, Libro 1. Madrid: La esfera de los libros.

Edwards, H. y Day, D. (2010). Marcas Pasión. Bogotá, D.C.: Panamericana.

Greene, R. (2011). Las 33 estrategias de la guerra. México DF: Océano.

Klaric, J. (2016). Estamos ciegos. Bogotá D.G.: Editorial Géminis.

Levitt, T. (1960). Marketing Myopia. Harvard Business Reviere, 45-56.

Loftus, G. (2010). Introducción. En G. Loftus, Lead like Ike (p. xvii). Nashville, Tennessee: Thomas Nelson Inc.

Lyons, E. (12 de julio de 2017). How to Harness Linking Value to Create Brand Affinity. Recuperado de www.ama.org: https://www.ama.org/publications/eNewsletters/ Marketing-News-Weekly/Pages/experiential-marketers-can-lasso-hidden-power-oflinking-value.aspx

Martínez, D. (2012). Zara: visón y estrategia de Amancio Ortega. Barcelona, Editorial Conecta. 
Moss, L. (10 de diciembre de 2013). Red Fox's Secret Hunting Weapon Revealed | MNN Mother Nature Network. Mother Nature Network. Recuperado de http://www.mnn. com/earth-matters/animals/stories/red-foxs-secret-hunting-weapon-revealed

National Geographic. (2010). Telarañas <tirachinas > para atrapar mosquitos. Recuperado de http://www.nationalgeographic.es/noticias/ara-a-tirachinas-noticia

Slywotzky, A. (2012). Nespresso y la demanda que por poco lo no es. En K. W. Adrián Slywotzky, Demanda: Crear lo que las personas desean antes de que sepan qué quieren (pp. 184207). Bogotá DC: Editorial Buena Semilla.

Taborsky, M. (2006). Ethology in a New Era. Ethology 112 The author journal compilation, $1-6$.

Tinbergen, N. (2005). Fascimile of on Aims and Methods of Ethology. Animal Biology, 55(4), 297-321. 